\title{
HD 165246: an early-type binary with a low mass ratio`
}

\author{
P. Mayer ${ }^{1}$, P. Harmanec ${ }^{1}$, and K. Pavlovski ${ }^{2}$ \\ 1 Astronomical Institute of the Charles University, Faculty of Mathematics and Physics, V Holešovičkách 2, 18000 Praha 8 , \\ Czech Republic \\ e-mail: mayer@cesnet.cz \\ 2 Department of Physics, Faculty of Science, University of Zagreb, Bijenička cesta 32, 10000 Zagreb, Croatia
}

Received 14 September 2012 / Accepted 27 November 2012

\section{ABSTRACT}

\begin{abstract}
Analyses of 13 FEROS spectra from the ESO archive and $617 \mathrm{~V}$-band photometric observations from the ASAS3 database allowed us to demonstrate that HD 165246 is a double-lined spectroscopic binary. As an earlier finding revealed, HD 165246 is also an eclipsing system. We were able to derive consistent orbital and light-curve solutions and all basic physical properties of the system. The period of this $\mathrm{O} 8 \mathrm{~V}+\mathrm{B} 7 \mathrm{~V}$ binary is 4.592706 and the semiamplitudes of the radial-velocity curves are $K_{1}=55.5 \mathrm{~km} \mathrm{~s}^{-1}$ and $K_{2}=321 \mathrm{~km} \mathrm{~s}^{-1}$. As the mass ratio is small (0.173), the secondary lines cannot be seen directly in the spectra; however the application of spectral disentangling allowed us to detect weak Balmer and He I lines of the secondary component. The primary component rotates with a high projected velocity of $v \sin i=243 \mathrm{~km} \mathrm{~s}^{-1}$. A combined radial-velocity and light-curve solution led to the component masses and radii expected for the young stars of the given spectral types. Due to the high rotation velocity, the primary component might display changes in surface abundances of some elements. However, we did not find any significant differences with respect to the abundances of slowly rotating stars.
\end{abstract}

Key words. stars: early-type - binaries: eclipsing - stars: individual: HD 165246

\section{Introduction}

Massive stars of spectral type $\mathrm{O}$ are astrophysically important in several aspects. Their evolution is fast and they affect the chemical composition of the circumstellar matter. Their accurate modelling via stellar-evolutionary models has to take into account a significant mass loss from these stars via stellar wind, a problem that has not yet been self-consistently solved. The most accurate way to obtain reliable physical properties of $\mathrm{O}$ stars is to study detached eclipsing binaries among them, though rare they are. Another problem of general importance is the formation of such massive stars. It was recently discussed by Chini et al. (2012), who found from their survey that the majority of known $\mathrm{O}$ stars form close binary systems with components of similar masses. The ratio of masses of binary components $q=m_{2} / m_{1}$ is a very important parameter; different theories of binary origin predict different mass ratio distributions. There might be an observational bias, however. Since O stars have very high luminosities, it is very hard to discover O-type binaries with a low mass ratio as the luminosity of the lighter component represents usually only a small fraction of the total luminosity of the system. In an earlier search, Garmany et al. (1980) found no case with $q<0.3$ among the early-type binaries. Nowadays, a few binaries with $q<0.3$ are known. Our literature survey revealed three massive binaries with (mutually similar) $q$ of about 0.24 :

- O 5 star HD 37022 ( $\theta 1$ Ori C); see Kraus et al. (2009),

- B7 Iab star HD 53975 (HR 2679); see Gies et al. (1994), and

- O6 V star HD 199579 (HR 8023); see Williams et al. (2001).

* Based on the spectra obtained with the European Southern Observatory telescopes and extracted from the ESO/ST-ECF Science Archive Facility, as well as on the $V$ photometry from the ASAS3 archive.
Here, we shall report the discovery that the $08 \mathrm{~V}$ binary HD 165246 has an even lower mass ratio and is therefore worth investigating in detail.

\section{Current knowledge about HD 165246}

The star HD $165246\left(\mathrm{CD}-24^{\circ} 13880 ; V=7.7\right)$ was classified O8 V(n) by Walborn (1972). It is a visual binary ADS 11049 (WDS 18061-2412, separation 1'.9, magnitude difference 4. 0 ) and a member of the O-type association Sgr OB1. There are several other binaries in this association: the eclipsing binaries V3903 Sgr (Vaz et al. 1997) and $\mu$ Sgr (Polidan \& Plavec 1984), as well as the spectroscopic binaries HD 164402 (Stickland \& Lloyd 1999), HD 164816 (Trepl et al. 2012), HD 165052 (Linder et al. 2007), and Herschel 36 (Arias et al. 2010). Otero (2007) discovered the eclipsing-binary nature of HD 165246 using the ASAS3 data (Pojmanski 2002) and derived a linear ephemeris

$T_{\text {Min.I }}=$ HJD $2452383.888+4.5928 \times E$.

The minima are quite narrow, with the depths of $0^{\mathrm{m}} \cdot 10$ and $0^{\mathrm{m}} \cdot 02$ in the $V$ band.

\section{Spectroscopy and preliminary orbital solutions}

In the ESO archive, there are 13 FEROS spectra taken by several observers in the years 2006 to 2009. The FEROS spectrograph works at the $2.2 \mathrm{~m} \mathrm{ESO/MPI} \mathrm{telescope} \mathrm{at} \mathrm{La} \mathrm{Silla.} \mathrm{The} \mathrm{spectra}$ have a resolving power of 48000 and cover the spectral region from 3575 to $9215 \AA$. They are available as pipeline products from the archive and are listed in Table 1. An example of the line profiles is in Fig. 1. There are also three spectra of this binary in the HST-STIS archive. They cover the wavelengths from 1160 
Table 1. RVs of six strong spectral lines of the primary of HD 165246 measured on the FEROS spectra in SPEFO.

\begin{tabular}{lcccccc}
\hline \hline $\begin{array}{l}\text { HJD } \\
-2400000\end{array}$ & $\begin{array}{c}\mathrm{H} \beta \\
\left(\mathrm{km} \mathrm{s}^{-1}\right)\end{array}$ & $\begin{array}{c}\mathrm{H} \gamma \\
\left(\mathrm{km} \mathrm{s}^{-1}\right)\end{array}$ & $\begin{array}{c}\text { He I 4026 } \\
\left(\mathrm{km} \mathrm{s}^{-1}\right)\end{array}$ & $\begin{array}{c}\mathrm{He} \mathrm{I} \mathrm{4471} \\
\left(\mathrm{km} \mathrm{s}^{-1}\right)\end{array}$ & $\begin{array}{c}\mathrm{He} \mathrm{II} \mathrm{4541} \\
\left(\mathrm{km} \mathrm{s}^{-1}\right)\end{array}$ & $\begin{array}{c}\text { He II 4686 } \\
\left(\mathrm{km} \mathrm{s}^{-1}\right)\end{array}$ \\
\hline 53965.5722 & -41.8 & -37.2 & -42.4 & -45.5 & -33.25 & -36.5 \\
53966.5548 & 13.7 & 18.7 & 4.8 & 9.7 & 28.90 & 30.5 \\
53967.5295 & 30.3 & 38.6 & 28.9 & 31.5 & 49.30 & 47.4 \\
53968.5284 & -26.8 & -21.7 & -35.0 & -32.9 & -19.70 & -18.5 \\
53969.5321 & -66.2 & -57.7 & -74.6 & -69.1 & -56.85 & -60.7 \\
53970.5551 & -20.7 & -16.7 & -26.7 & -25.1 & -15.00 & -14.3 \\
54209.9196 & 10.4 & 16.9 & 2.1 & 1.7 & 24.25 & 23.5 \\
54211.9021 & -27.9 & -25.4 & -38.1 & -35.3 & -21.00 & -22.7 \\
54246.8379 & 22.0 & 26.2 & 15.5 & 19.4 & 35.55 & 32.0 \\
54602.8005 & -54.0 & -49.0 & -62.2 & -61.5 & -50.70 & -50.9 \\
54626.7012 & -57.9 & -49.0 & -64.5 & -62.1 & -46.25 & -54.0 \\
54955.9289 & -29.0 & -26.0 & -37.9 & -37.7 & -17.75 & -24.4 \\
54956.9261 & -66.2 & -60.2 & -71.2 & -69.5 & -58.75 & -57.6 \\
\hline
\end{tabular}

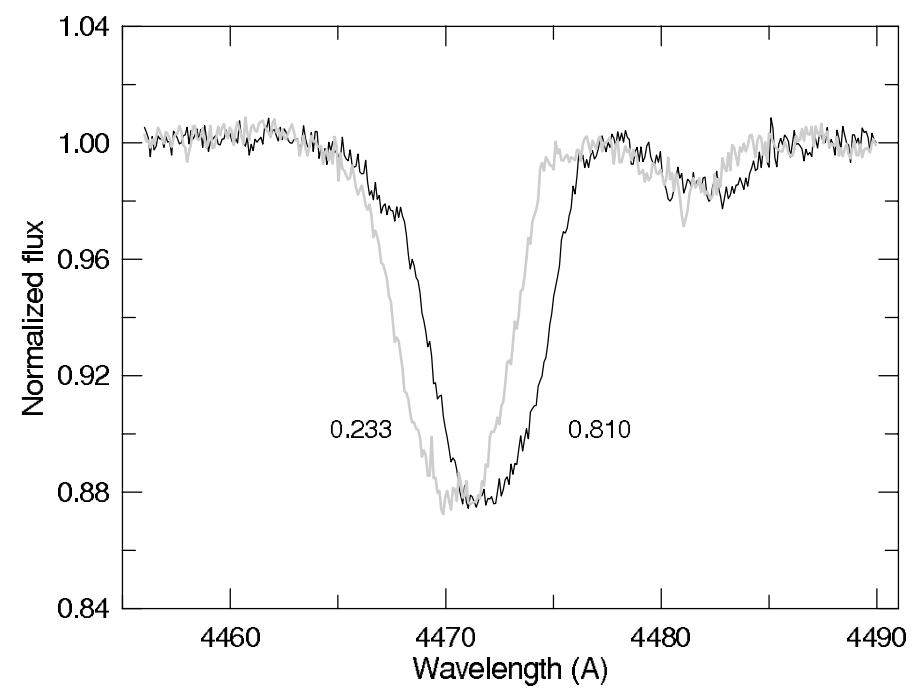

Fig. 1. Line profiles of the He I $\lambda 4471$ line at phases close to quadratures.

to $1360 \AA$ A. However, due to short spectrograph echelle orders and the wide lines of the $\mathrm{O} 8$ star, the radial velocities (RVs), which we tried to determine from them, turned out unreliable and are not used here.

The FEROS spectra agree perfectly with the O8 V classification. We first measured the RVs using several He I lines ( $\lambda \lambda 4026$ and 4471), He II lines ( $\lambda \lambda 4541$ and 4686), and Balmer $\mathrm{H} \beta$ and $\mathrm{H} \gamma$ with the programme SPEFO (Horn et al. 1996; Škoda 1996), comparing the direct and flipped line profiles on the computer screen. The measured RVs are in Table 1.

The lines are quite wide, the projected rotation velocity being $v \sin i=243 \mathrm{~km} \mathrm{~s}^{-1}$ (see later). The period and the conjunction time of the RV curve agree well with Otero's ephemeris (Eq. (1)). The parameters of the RV curve can be determined with good accuracy. However, the small RV amplitude in the eclipsing binary is rather surprising: in the He II lines $\lambda 4541$ and $\lambda 4686$, it is only $\approx 56 \mathrm{~km} \mathrm{~s}^{-1}$. No indications of the secondary lines are visible in the spectra (see lower panel in Fig. 3). Although the number of spectra is small and the photometry has only a modest signal-to-noise ratio $(\mathrm{S} / \mathrm{N})$, the phase distribution of the spectra is good and the orbit is well defined. To check whether a small orbital eccentricity is present, we derived trial orbital solutions using the programme SPEL ${ }^{1}$. This programme carries out automatically the test on the statistical significance of the orbital eccentricity suggested by Lucy \& Sweeney (1971). The orbital solutions are summarized in Table 2 . We note that the most reliably measurable lines all indicate a small, but significant orbital eccentricity. It is also seen that both the semiamplitudes and the systemic velocities differ from line to line. While the differences in the systemic velocities can be explained by the line blending of rotationally broadened lines, the differences in the semiamplitudes could be indicative of the presence of weak lines of the secondary. For that reason we prefer estimating the mass function from the mean semiamplitude of the He II $\lambda 4541$ and $\lambda 4686$ lines, which should be only present in the spectrum of the primary. To this end, we used the mean RVs of both lines to derive another solution, also listed at the end of Table 2. It gives $K_{1}=55.9 \pm 1.0 \mathrm{~km} \mathrm{~s}^{-1}$, therefore a low mass function of $f(m)=0.0827 M_{\odot}$. Due to the presence of the eclipses and large rotation velocity, the inclination has to be large and the mass function can be satisfied only by a small mass ratio of about 0.17 .

We note that the visual component (see Introduction) should not affect our analysis. It should be out of the FEROS fiber (diameter $2^{\prime \prime}$ ). In a trial light-curve solution (see Sect. 4), we tried to assume $2 \%$ of the third light. However, because the results were the same as with the third-light contribution set to zero, we have not considered the third light in the final solution. It is interesting to note that there are three components in the interstellar $\mathrm{NaD}$ lines, with velocities $-52,-25$, and $-7 \mathrm{~km} \mathrm{~s}^{-1}$. According to the spectra in the ESO archive, while both more positive components are present in the spectra of HD 165052, HD 164794, 9 Sgr, and V3903 Sgr, the most negative component is only seen in the spectra of HD 165246. In the $\mathrm{H} \alpha$ line, there is an emission peak at $\approx 0 \mathrm{~km} \mathrm{~s}^{-1}$, rising to some $5 \%$ of the continuum level and with $F W H M \approx 0.7 \AA$. It obviously originates from the nebular emission of the $\mathrm{H}$ II region.

\section{The light-curve solution}

In the next step, we attempted to see how well the most reliable RVs based on the two He II lines can be reconciled with the available $V$-magnitude light curve. We extracted the $V$-band observations from the ASAS3 archive, using the data obtained with

1 The programme SPEL was written by the late Dr. J. Horn and never published. It was used in a number of publications, however, cf. Nemravová et al. (2012) and references therein. 
Table 2. Preliminary orbital solutions on RVs of several spectral lines measured directly in SPEFO.

\begin{tabular}{lccccccc}
\hline \hline Spectral line & $T_{\min . I}$ & $e$ & $\omega(\mathrm{deg})$ & $K_{1}\left(\mathrm{~km} \mathrm{~s}^{-1}\right)$ & $V_{\gamma}\left(\mathrm{km} \mathrm{s}^{-1}\right)$ & $\mathrm{rms}\left(\mathrm{km} \mathrm{s}^{-1}\right)$ & $p$ \\
\hline$\lambda 4026$ & $383.8 \pm 0.6$ & $0.084 \pm 0.034$ & $77.5 \pm 26.5$ & $51.4 \pm 1.9$ & $-21.9 \pm 1.2$ & 3.28 & 0.11 \\
$\lambda 4471$ & $383.8 \pm 0.6$ & $0.080 \pm 0.023$ & $55.9 \pm 24.7$ & $52.1 \pm 1.5$ & $-19.8 \pm 0.9$ & 2.64 & 0.04 \\
$\lambda 4541$ & $383.8 \pm 0.4$ & $0.066 \pm 0.013$ & $54.9 \pm 16.0$ & $55.8 \pm 0.9$ & $-4.3 \pm 0.6$ & 1.56 & 0.00 \\
$\lambda 4686$ & $383.8 \pm 0.8$ & $0.050 \pm 0.023$ & $33.7 \pm 34.3$ & $56.0 \pm 1.5$ & $-6.4 \pm 0.9$ & 2.66 & 0.17 \\
$\mathrm{H} \gamma$ & $383.9 \pm 0.6$ & $0.053 \pm 0.015$ & $51.7 \pm 23.2$ & $50.0 \pm 0.9$ & $-9.9 \pm 0.6$ & 1.64 & 0.04 \\
$\mathrm{H} \beta$ & $383.9 \pm 1.2$ & $0.023 \pm 0.021$ & $107.3 \pm 47.8$ & $49.5 \pm 1.0$ & $-15.9 \pm 0.6$ & 1.85 & 0.57 \\
\hline$\lambda 4541 \& 4686$ & 383.80 .5 & $0.057 \pm 0.014$ & $45.1 \pm 20.2$ & $55.9 \pm 1.0$ & $-5.3 \pm 0.6$ & 1.72 & 0.02 \\
\hline
\end{tabular}

Notes. The "rms" column gives the rms of 1 observation, epochs are in HJD-2452 000.0, and $p$ is the probability that the eccentricity $e$ is false according to the Lucy \& Sweeney (1971) test (the values smaller than 0.05 denote real eccentricity).

the diaphragm giving the lowest mean rms error (diaphragm 4 in this case). We were able to use a more extended dataset than that available to Otero (2007). Omitting both observations with large rms errors and observations strongly deviating from the light curve, we ended with 617 observations covering the time interval JD 2452 140-2455037.

Since the O8V spectral type of the primary seems to be reliable and consistent with all available pieces of information, we decided to assume a normal mass and theoretical $T_{\text {eff }}$ for this star after Martins et al. (2005, their Table 1): $T_{\text {eff } 1}=33400 \mathrm{~K}$ and $M_{1}=21.95 M_{\odot}$. For the light- and the primary RV-curve solution we used the programme PHOEBE (Prša \& Zwitter 2005, 2006). We had to proceed in an iterative way. We started with the mass ratio of 0.175 , estimated from the mass function and the above mass of the primary and the projected semimajor axis of the binary orbit, both fixed at values that follow from the orbital solution for the mean RVs of the two He II lines (cf. Table 2). A preliminary solution showed that the primary is rotating $\approx 3$ times faster than what would correspond to the spinorbit synchronisation, so the ratio 3 was used in all trial PHOEBE solutions.

It soon turned out that the nearly symmetrically placed secondary minimum of the light curve can only be reconciled with the values of the longitude of periastron $\omega$ near $90^{\circ}$ and that the $\mathrm{RV}$ curve of the primary shows a measurable rotational effect, which was recorded in two observed RVs from appropriate orbital phases. Since the programme SPEL is not modelling the rotational effect, we had to improve the semiamplitude of the primary RV curve from the PHOEBE fit and also iteratively improve the exact value of the mass function. The final solution is summarized in Table 3 and the corresponding RV and $V$ light curves are shown in Fig. 2.

Although the $\mathrm{S} / \mathrm{N}$ of the light curve is moderate with respect to relatively shallow minima, the resulting radius of the primary, $7.35 R_{\odot}$, is quite typical for an $\mathrm{O} 8 \mathrm{~V}$ star (compare e.g., Harmanec 1988). The radius of $2.11 R_{\odot}$ appears a bit too small for the mass of the secondary. The projected synchronous rotational velocity of the primary component should be $81 \mathrm{~km} \mathrm{~s}^{-1}$, which is three times smaller than the observed one of $243 \mathrm{~km} \mathrm{~s}^{-1}$. The PHOEBE solution resulted in an improved linear ephemeris

$$
T_{\text {Min. } I}=\text { HJD } 2452383.8927(45)+4.592706(14) \times E .
$$

It is encouraging to see that a rather pronounced rotational effect predicted by the PHOEBE solution is confirmed by the observed RVs. We would like to point out, however, that the reality of the orbital eccentricity should be investigated again when more numerous RVs and more accurate photometry are available. Although the eccentricity is real by the Lucy \& Sweeney (1971)
Table 3. Parameters of the binary HD 165246.

\begin{tabular}{lc}
\hline \hline Parameter & Value \\
\hline Period (days) & $4.592706(14)$ \\
$T_{\text {periastr. } I}$ & HJD 2 452 383.8831(45) \\
$T_{\text {min. } I}$ & HJD 2 $452383.8927(45)$ \\
$e$ & $0.0459(31)$ \\
$\omega\left({ }^{\circ}\right)$ & $89.2(2.1)$ \\
inclination $\left(^{\circ}\right)$ & $83.64(24)$ \\
$\Omega_{1}$ & $5.033(65)$ \\
$\Omega_{2}$ & $4.281(49)$ \\
$T_{\text {eff }}(\mathrm{K})$ & $12530(1000)$ \\
$R_{1}\left(R_{\odot}\right)$ & $7.35(16)$ \\
$R_{2}\left(R_{\odot}\right)$ & $2.11(5)$ \\
$a\left(R_{\odot}\right)$ & $34.32(59)$ \\
$M_{1}\left(M_{\odot}\right)$ & $21.95($ fixed $)$ \\
$M_{2}\left(M_{\odot}\right)$ & $3.80(7)$ \\
$\log g$ [cgs] & $4.05(2)$ \\
$\log g_{2}[\mathrm{cgs}]$ & $4.37(2)$ \\
$L_{1}\left(L_{1}+L_{2}=1\right)$ & $0.986(2)$ \\
$L_{2}\left(L_{1}+L_{2}=1\right)$ & $0.014(2)$ \\
\hline
\end{tabular}

Notes. The numbers in parenthesis are the mean errors (m.e.) as given by PHOEBE; in some cases, the errors were calculated from the PHOEBE m.e.

test, the longitude of periastron near $90^{\circ}$ might indicate that the eccentricity is spurious, caused by the nonspherical shape of the primary (see Harmanec 2003, for a more detailed discussion).

\section{The KOREL disentangling}

Since the 13 FEROS spectra are well distributed in the orbital phase, we decided to apply the disentangling technique; in the first step we used the programme KOREL (Hadrava 1995, 1997, 2004, 2005). We analysed the parts of the FEROS spectra covering the wavelength range from 4000 to $5100 \AA$. In particular, we formed three subsets in the neighbourhood of the Balmer lines $\mathrm{H} \delta, \mathrm{H} \gamma$, and $\mathrm{H} \beta$ (4000-4175 $\AA, 4310-4400 \AA$, and 4800-4945 $\AA$ ) and combined them into one KOREL input dataset. Similarly, we formed two subsets containing the neighbourhood of the He II $\lambda 4541$ and $\lambda 4686$ lines (4495-4565 and 4670-4747 $\AA$ ) and also combined them into another KOREL input dataset.

To see whether KOREL is able to detect weak lines of the secondary with some confidence, we first used the combined dataset containing all five subsets. Keeping the semiamplitude of the primary (which is well constrained by the direct RV measurements), eccentricity, and $\omega$ fixed at values from the PHOEBE solution, we investigated the run of the sum of squares of residuals as a function of the semiamplitude of the secondary $K_{2}$. 

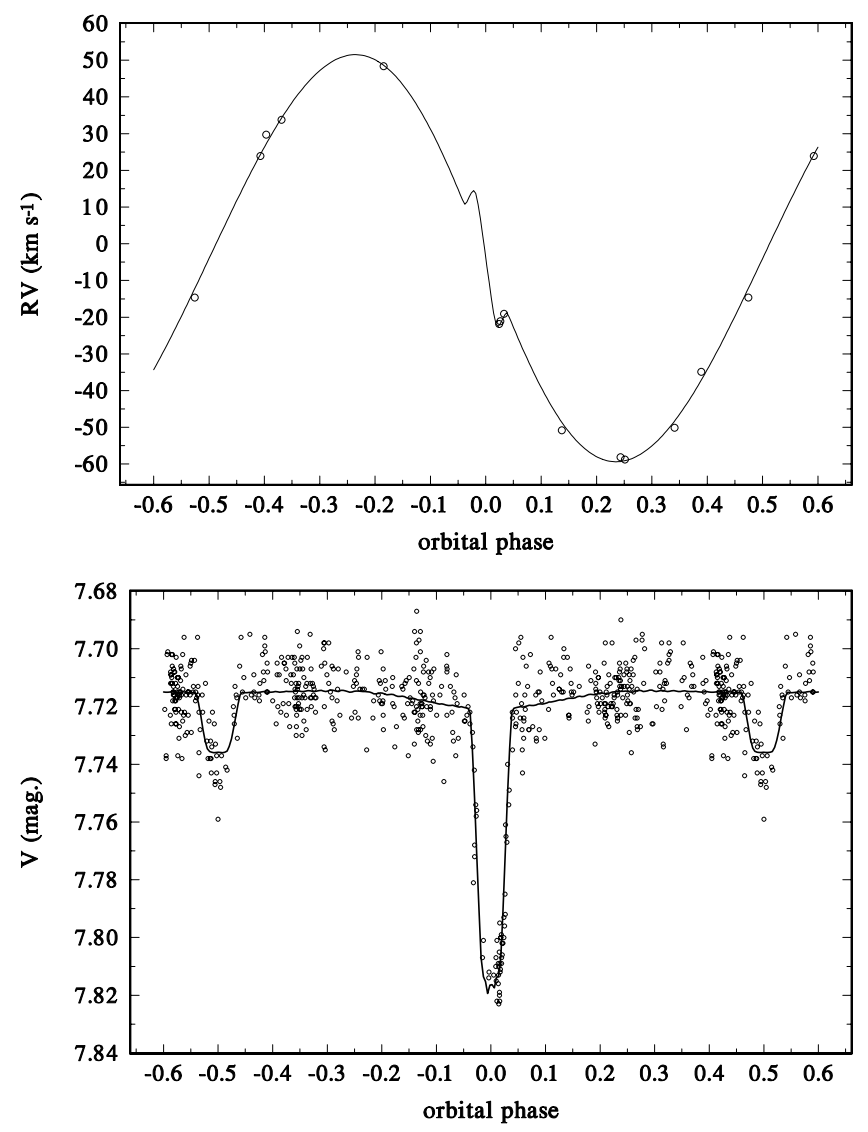

Fig. 2. The RV curve of the primary based on the mean RVs of the He II lines (top) and the $V$ light curve based on the ASAS3 data (bottom) compared with the final PHOEBE solution shown by solid lines.

Regrettably, we could not find a trustworthy solution this way. This is not so surprising if one realizes that according to the PHOEBE solution, the secondary is for full 4.6 fainter than the primary.

We therefore fixed all elements including the mass ratio of 0.173 from the PHOEBE solution and used KOREL only to disentangle the individual spectra. The programme KOREL returned very weak lines of the secondary for $\mathrm{H} \mathrm{I}$ and $\mathrm{He} \mathrm{I}$ lines and flat continua for the He II lines. To the best of our knowledge, this goes beyond the current "record" by Holmgren et al. (1999), who detected a secondary for 4.0 fainter than the primary in AR Cas. Two spectral segments of the disentangled spectra are shown in Fig. 3.

\section{Spectroscopic analysis}

\subsection{Effective temperatures}

The condition that the sum of the light factors, a relative contribution of the components to the total light of the system, is equal to unity constrains the search for the optimal parameters (Tamajo et al. 2011). In a new release of the computer programme for the constrained optimal fitting of disentangled component spectra, the following parameters could be adjusted for both components: the effective temperature, the surface gravity, the light dilution factor, Doppler shift, the projected rotational velocity, and the vertical shift to adjust for the continuum (in spectral disentangling, the disentangled component spectra are shifted and an additive factor should be applied to return them to the continuum level, cf. Pavlovski \& Hensberge 2005). The comprehensive
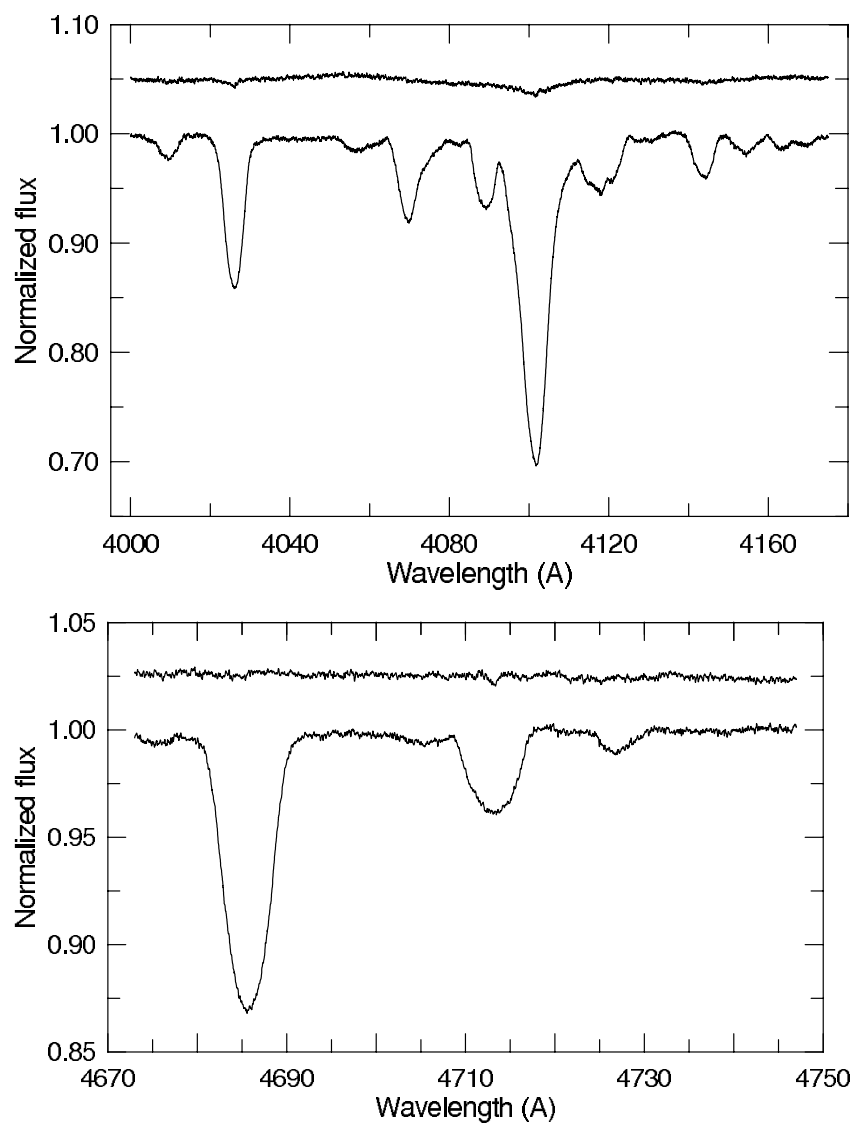

Fig. 3. Upper panel: the disentangled spectra of HD 165246 around $\mathrm{H} \delta$. The secondary spectrum is shifted by +0.05 . Note the presence of $\mathrm{He}$ I line $\lambda 4026$ and $\mathrm{H} \delta$ in the secondary spectrum. Lower panel: the disentangled spectra around the He II line $\lambda 4686$. The secondary spectrum is shifted by +0.025 . The He II line is absent in the secondary spectrum.

grids OSTAR and BSTAR of theoretical spectra calculated by Lanz \& Hubeny $(2003,2007)$, were implemented for the optimal fitting. For the optimisation, a genetic algorithm is used (Charbonneau 1995). Further description of the code is given in Pavlovski et al. (2012, in prep.).

Only the hydrogen Balmer lines, $\mathrm{H} \delta, \mathrm{H} \gamma$, and $\mathrm{H} \beta$ are selected for the optimal fitting. Due to the diffuse interstellar band centred on $\lambda 4882$ (Herbig 1995) and affecting almost the whole red wing of $\mathrm{H} \beta$, this line is only partially used. In fitting the line profiles of $\mathrm{H} \delta$ and $\mathrm{H} \gamma$, the blends due to various metal lines are also excluded. The surface gravities and light factors of both components were fixed at the values of the solution of Table 3 .

Since the Balmer lines are almost insensitive to the rotational broadening with the exception of the very core of the lines, the projected rotational velocity was also fixed in these calculations. The projected rotational velocity was derived from the isolated helium lines in an iterative cycle by determining the effective temperatures from the Balmer lines. We arrived at $v \sin i=242.6 \pm 2.7 \mathrm{~km} \mathrm{~s}^{-1}$. A strong correlation of $T_{\mathrm{eff}}$ and $\log g$ occurs when fitting the Balmer line profiles. It is the major limitation in choosing the optimal model atmosphere and for subsequent abundance analysis. Fortunately, it is possible to derive accurate values of $\log g$ for both binary components from the combined light and velocity curve analysis for the eclipsing and double-lined spectroscopic binaries. Thanks to this, the degeneracy in $T_{\text {eff }}$ and $\log g$ can be lifted. In the present analysis, we therefore also fixed $\log g$ for both components at the values of Table 3. 


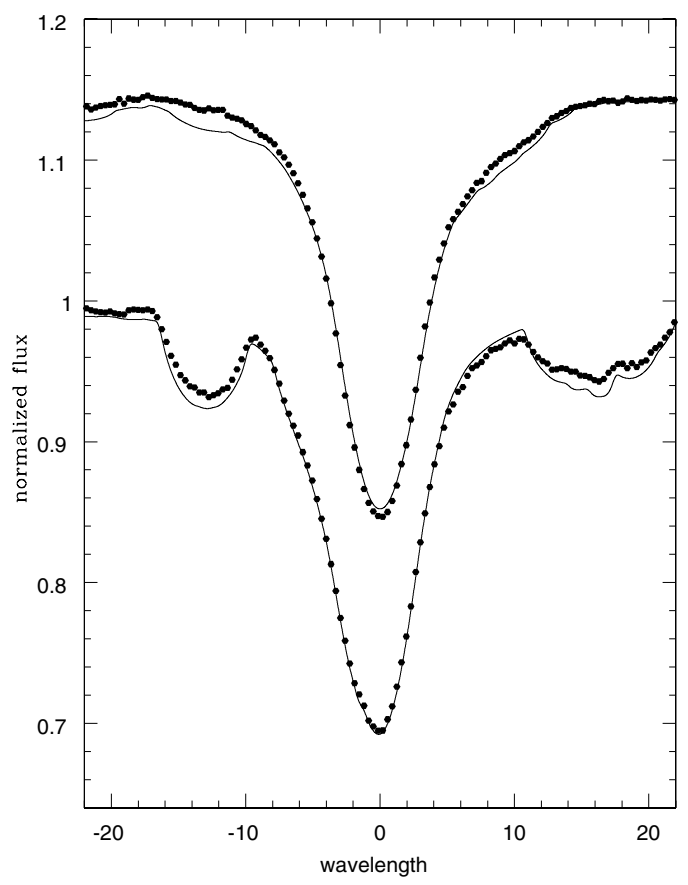

Fig. 4. A comparison of the disentangled and fitted line profiles of the $\mathrm{H} \gamma$ (top) and $\mathrm{H} \delta$ (bottom) Balmer lines of the primary. Renormalised disentangled spectra are shown by black dots, while the best-fitting synthetic line profiles derived via optimisation in the OSTAR grid of nonLTE spectra calculated by Lanz \& Hubeny (2003) are shown as solid lines. Blends conspicuous on hydrogen line profiles due to the various metals are not fitted. Theoretical spectra calculated for the solar abundance were used.

Hence, the only free parameters that were optimized were the effective temperatures, Doppler shifts, and the vertical shifts. The mean values of the effective temperatures derived in these calculations individually for the three Balmer lines studied are $T_{\text {eff }}=33300 \pm 400 \mathrm{~K}$ and $T_{\text {eff }}=15800 \pm 700 \mathrm{~K}$ for the primary and secondary, respectively. The quality of the fits for the $\mathrm{H} \delta$ and $\mathrm{H} \gamma$ lines can be judged from Fig. 4 .

\subsection{Abundances}

Theoretical spectra of the components were calculated in a "hybrid" approach with local thermodynamic equilibrium (LTE) atmospheres and non-LTE line formation (Nieva \& Przybilla 2007). Model atmospheres were calculated with the ATLAS9 code. Non-LTE level populations and model spectra were obtained with DETAIL and SURFACE (Giddings 1981; Butler \& Giddings 1985). Non-LTE-level populations and the synthetic spectra of $\mathrm{H}, \mathrm{He}, \mathrm{C}, \mathrm{N}, \mathrm{O}, \mathrm{Mg}$, and $\mathrm{Si}$ elements were computed using the most recent model atoms (cf. Table 3 in Nieva \& Przybilla 2012).

The projected rotational velocity of the primary is rather high and the lines of metals are broad and shallow. The typical S/N of the input spectra is $\sim 250$, but the disentangling process enhances the $\mathrm{S} / \mathrm{N}$ of disentangled individual spectra of the components because it works as a co-addition. Since the primary contributes more than $98 \%$ of the total light of the system, its disentangled spectrum gains about 3.5 times in the $\mathrm{S} / \mathrm{N}$, which means its disentangled spectrum has an $S / N \sim 500$. Therefore, in spite of broad and shallow metal lines that overlap and produce complex blends and spectral features, fine details can still be studied. We were able to trace spectral features down to $0.3 \%$ of the continuum level with confidence.
Table 4. Abundance of helium derived from several He I lines.

\begin{tabular}{lcc}
\hline \hline Line $(\AA)$ & Transition & $\epsilon(\mathrm{He})$ \\
\hline 4471.5 & 1s2p-1s4d (t) & $0.089 \pm 0.005$ \\
4921.9 & 1s2p-1s4d (s) & $0.088 \pm 0.004$ \\
5015.7 & 1s2s-1s3p (s) & $0.098 \pm 0.006$ \\
5047.7 & $1 \mathrm{~s} 2 \mathrm{p}-1 \mathrm{~s} 4 \mathrm{~s}(\mathrm{~s})$ & $0.085 \pm 0.004$ \\
\hline Robust mean & & $0.089 \pm 0.003$ \\
\hline
\end{tabular}

Table 5. Abundances for the primary component of HD 165246.

\begin{tabular}{cccc}
\hline \hline Elem. & HD 165246 A & B-type & Sun \\
\hline $\mathrm{He}$ & $10.95 \pm 0.02$ & $10.99 \pm 0.01$ & $10.98 \pm 0.01$ \\
$\mathrm{C}$ & $8.27 \pm 0.04$ & $8.33 \pm 0.04$ & $8.47 \pm 0.05$ \\
$\mathrm{~N}$ & $7.75 \pm 0.07$ & $7.79 \pm 0.04$ & $7.87 \pm 0.05$ \\
$\mathrm{O}$ & $8.48 \pm 0.06$ & $8.76 \pm 0.05$ & $8.73 \pm 0.05$ \\
$\mathrm{Mg}$ & $7.44 \pm 0.10$ & $7.56 \pm 0.05$ & $7.64 \pm 0.04$ \\
$\mathrm{Si}$ & $7.48 \pm 0.07$ & $7.50 \pm 0.05$ & $7.55 \pm 0.04$ \\
{$[\mathrm{~N} / \mathrm{C}]$} & $-0.52 \pm 0.08$ & $-0.54 \pm 0.06$ & $-0.60 \pm 0.07$ \\
{$[\mathrm{~N} / \mathrm{O}]$} & $-0.73 \pm 0.09$ & $-0.97 \pm 0.06$ & $-0.86 \pm 0.07$ \\
\hline
\end{tabular}

Notes. The cosmic abundance pattern derived by Nieva \& Przybilla (2012) and the standard solar abundance by Asplund et al. (2009) are given in Cols. 3 and 4, respectively.

First, we estimated the microturbulent velocity. For hot, massive stars, oxygen and/or silicon lines serve this purpose since they are well populated in their spectra. In the present work, we have only used the silicon lines, neglecting the oxygen lines that occur only in complex blends.

Silicon is present in two ionisation stages, with the Si IV lines being much stronger than those of Si III. Some of them are blended like Si IV $\lambda 4088.9$ with oxygen lines and Si IV $\lambda 4631.2$ with the carbon and oxygen lines. Yet, they are still strong enough to permit the abundance estimates. Minimizing the scatter in the abundances from different silicon lines, we obtained the microturbulent velocity $\xi_{\mathrm{t}}=5 \pm 1 \mathrm{~km} \mathrm{~s}^{-1}$. It eventually resulted in the abundance of silicon $\log \epsilon_{\mathrm{Si}}=7.48 \pm 0.08$, in excellent agreement with recent comprehensive abundance studies of sharp-lined early B-type stars, $\log \epsilon_{\mathrm{Si}}=7.51 \pm 0.03$ by Simón-Diaz (2010), $\log \epsilon_{\mathrm{Si}}=7.50 \pm 0.05$ by Nieva \& Przybilla (2012), and the Sun $\log \epsilon_{\mathrm{Si}}=7.51 \pm 0.03$ (Asplund et al. 2009).

In accordance with the effective temperature of the primary, the helium lines are prominent in the optical spectrum and appear in two ionisation stages, He I and He II. The strengths of He I lines correspond to the $T_{\text {eff }}$ of the primary, while the strengths of the synthetic He II lines are too small in comparison to the disentangled line profiles. To obtain a satisfactory match, we would need to increase the model $T_{\text {eff }}$ by about $1500 \mathrm{~K}$. We have no clear explanation for this discrepancy, which is also found for the spectra from the Lanz \& Hubeny (2003) grid.

Table 4 contains the result of the helium abundance determination for the primary component from the most reliable He I lines. From the calculation point of view, the most trustworthy are the lines He I $\lambda 4471$ and $\lambda 4920$. The mean helium abundance for the primary component from the measured helium lines (Table 5) is $\epsilon_{\mathrm{He}}=0.090 \pm 0.010$ or $\epsilon_{\mathrm{He}}=0.089 \pm 0.006$ if only the two most reliable lines (He I $\lambda 4471$ and 14922 ) are considered. The quality of the fit for the studied He I lines can be judged from Fig. 5.

Abundances of other elements are estimated from the fitting of complex profiles. In Fig. 6, such a complex blend of O II, $\mathrm{C}$ III, and $\mathrm{Si}$ IV in the blue wing of $\mathrm{H} \delta$ line is shown. The final 

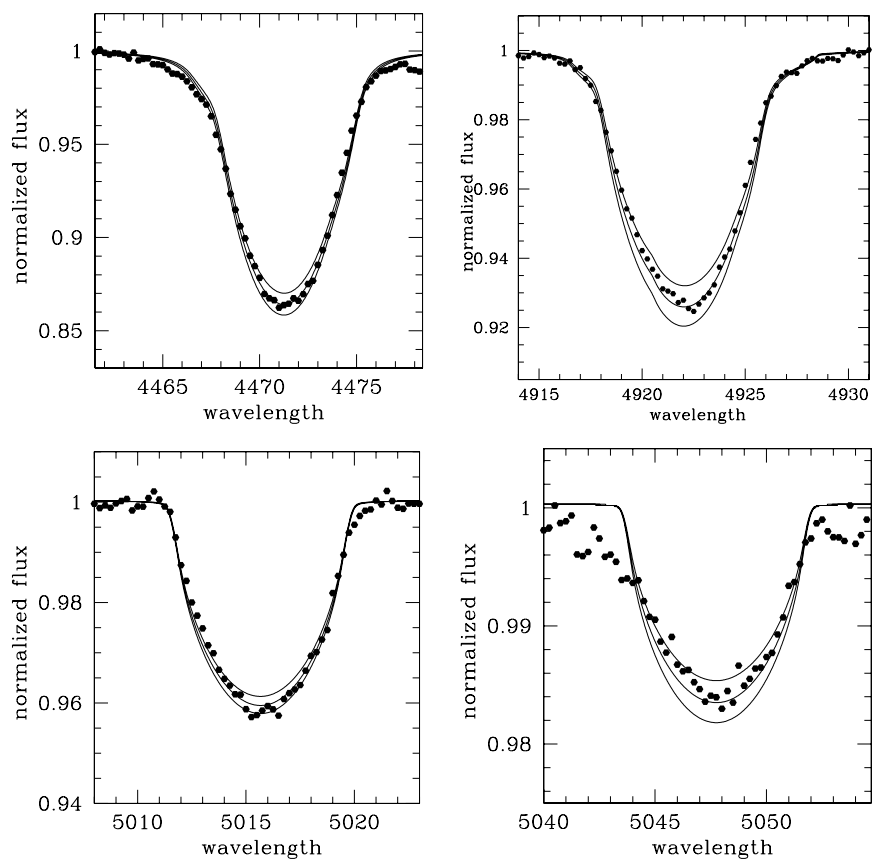

Fig. 5. Theoretical spectra of $\mathrm{He}$ I lines calculated for the atmospheric parameters of the primary component $\left(T_{\text {eff }}=33300 \mathrm{~K}, \log g=3.97\right.$, $\left.\xi=5 \mathrm{~km} \mathrm{~s}^{-1}\right)$ and three different abundances of helium $(\epsilon(\mathrm{He})=0.08$, 0.09 and 0.10 ) (solid lines) compared to the renormalised disentangled profiles of the helium lines of the primary component (black dots).

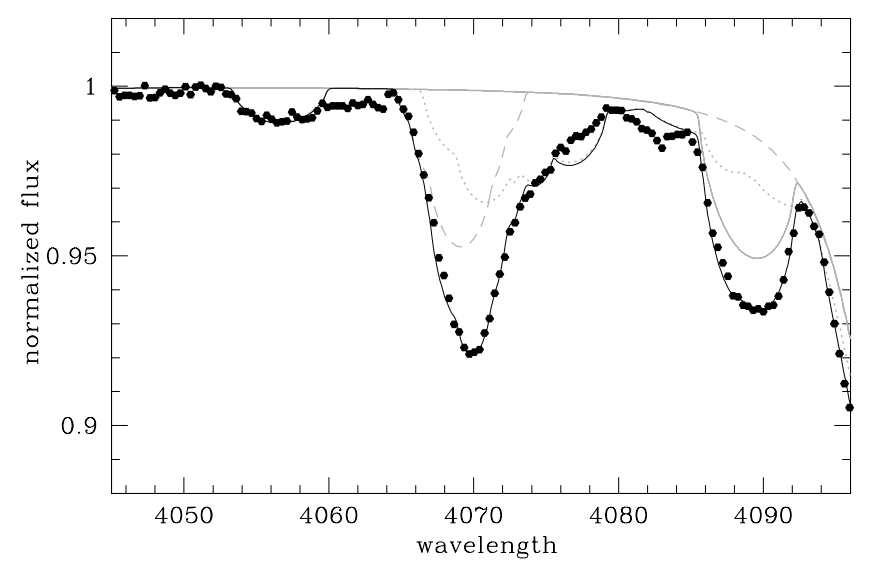

Fig. 6. Complex blends in the blue wing of $\mathrm{H} \delta$ line including lines of O II (dotted), C III (dashed), and Si IV (light grey solid). The composite spectrum for the abundances given in Table 4 represents the solid dark grey line. The renormalised, disentangled primary's spectrum is shown by black dots.

results are summarized in Table 5 and discussed in the following section.

\section{Discussion}

In Sect. 4, we found the fundamental quantities of the components of the binary system HD 165246. The more luminous component turned to be a high-mass star with $M \sim 22 M_{\odot}, R=$ $7.4 \pm 0.2 R_{\odot}$, and a high rotational velocity $v=244 \pm 3 \mathrm{~km} \mathrm{~s}^{-1}$. Therefore, this star is suitable for probing the predictive power of a new generation of stellar evolutionary models, with the rotation effects included in the calculations. Unfortunately, our analysis is hampered by the barely visible secondary's spectrum and the rather limited number of available spectra. In conjunction with

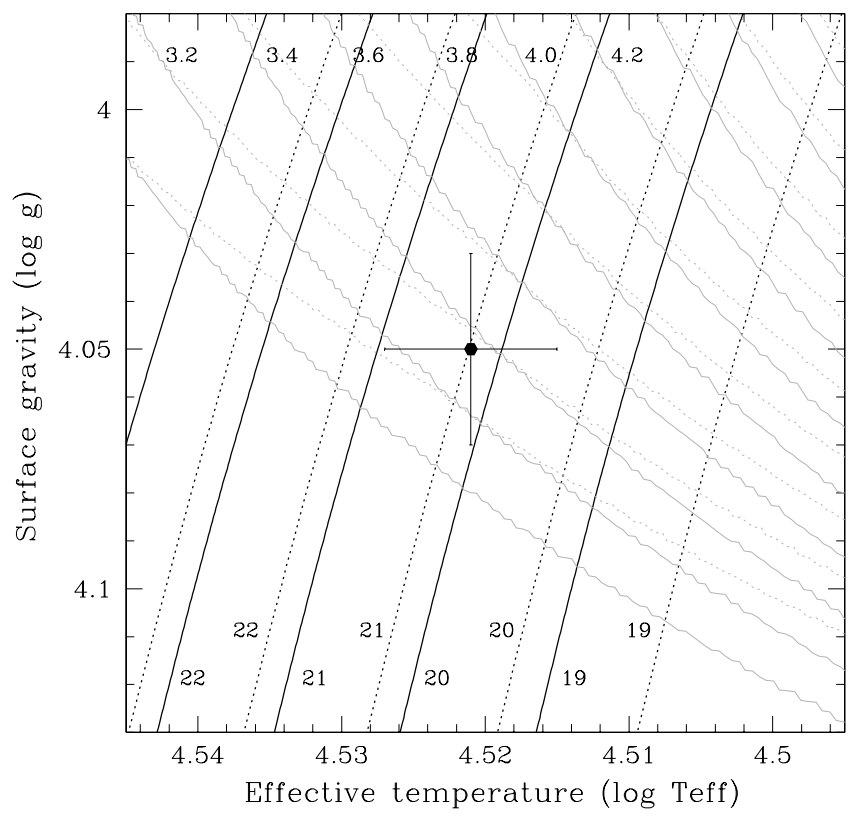

Fig. 7. Location of the primary component of HD 165246 in $T_{\text {eff }}$ vs. $\log g$ plane. Evolutionary tracks for models are labelled by their mass in the solar units. Evolutionary models by Brott et al. (2011) for the initial rotational velocities of $v=0$ and $270 \mathrm{~km} \mathrm{~s}^{-1}$ are plotted as solid and dotted lines, respectively. Isochrones are plotted as solid and dotted thin lines.

all-sky photometry, these were a limiting factor in matching the observations to the theoretical predictions.

In Fig. 7, the location of the primary component of HD 165246 in the $\log g-T_{\text {eff }}$ plane is compared to the evolutionary calculations of the Utrecht group (Brott et al. 2011). The location of the primary corresponds to a mass about $1 M_{\odot}$ smaller than we assumed; nevertheless, it is an acceptable result. This is encouraging, but it still does not permit discrimination between the rotating and non-rotating evolutionary models, mainly because of the strong degeneracy of the models with respect to the initial rotational velocity. Another observational constraint is needed to distinguish between them. Since model calculations predict the most pronounced changes in nitrogen abundance, it is hoped that nitrogen can serve as another observable and eventually break down the degeneracy in the evolutionary tracks with respect to the initial rotational velocities.

From isochrones we estimated the age of the primary component $\tau \sim 3.3 \pm 0.2 \mathrm{Myr}$ with almost no difference between non-rotating and rotating evolutionary models, with the initial rotational velocities $v \sin i=0$ and $270 \mathrm{~km} \mathrm{~s}^{-1}$.

Using the formulae by Zahn $(1975,1977)$ for the synchronization and circularization times, one gets 39 and $330 \mathrm{My}$, respectively. The reason why these times are so long is the small mass ratio. Therefore, the fast rotation is not surprising.

Rotating evolutionary models predict changes in the surface abundance pattern. Inspection of the Utrecht models for the mass of $20 M_{\odot}$ reveals that for the estimated age of the binary, $\tau \sim 3$ Myr, the rotational velocity has not yet changed from its initial rotational velocity. Therefore, we consider the model with initial rotational velocity $271 \mathrm{~km} \mathrm{~s}^{-1}$ as closest to the measured primary's rotational velocity, $v \sim 244 \mathrm{~km} \mathrm{~s}^{-1}$. When compared with the initial chemical composition, we found that the model predicts the helium and nitrogen enrichment by 0.005 and 0.282 dex, respectively, and a depletion of carbon and oxygen by 0.089 and 0.017 dex, respectively. It is obvious that the change 
in the nitrogen abundance due to the rotational mixing should be detectable within the accuracy of the present abundance determination. The most robust indicator of the abundance change is the ratio of the nitrogen to carbon abundances, $[\mathrm{N} / \mathrm{C}]$. The model calculations predict changes from the initial $[\mathrm{N} / \mathrm{C}]=-0.49$ to $[\mathrm{N} / \mathrm{C}]=-0.12$ for the age of $4 \mathrm{Myr}$. Our abundance estimates for the primary give $[\mathrm{N} / \mathrm{C}]=-0.52 \pm 0.08$; hence, no changes in the abundances are detected. This result supports the previous findings for the high-mass stars in close binaries (Pavlovski \& Southworth 2009; Pavlovski et al. 2009).

Abundances we derived in the present work for the primary component of HD 165246 (the second column in Table 5) are compatible with the cosmic abundance pattern of Nieva \& Przybilla (2012) within their errors, except for the oxygen abundance. Further work on the larger sample of the stars in close binaries is needed to clarify this discrepancy.

We have found that the binary system HD 165246 contains a rapidly rotating high-mass star at an early stage of evolution. This star is an ideal object for probing the predictions of rotating evolutionary models. It is also an important object for the theory of binary-star origin since it is believed that the majority of the $\mathrm{O}$-star binaries occurs in pairs with a mass ratio close to one (see, e.g., Chini et al. 2012, for details).

We therefore encourage both the more numerous high $\mathrm{S} / \mathrm{N}$ and high-spectral-resolution spectroscopic observations and accurate multicolour photometry. Since HD 165246 is a relatively bright target, such observations could be obtained even with moderate-size telescopes.

Acknowledgements. We thank an anonymous referee, whose comments were very helpful. We profited from the use of the computerized bibliography maintained in the NASA/ADS system and the CDS in Strasbourg. The Czech authors were supported by the grant P209/10/0715 of the Czech Science Foundation and also by the research programme MSM0021620860. K.P. acknowledges support from the Croatian MZOS under research grant 119-0000000-3135.

\section{References}

Arias, J. I., Barbá, R. H., Gamen, R. C., et al. 2010, ApJ, 710, L30

Asplund, M., Grevesse, N., Sauval, J., \& Scott, P. 2009, ARA\&A, 47, 481

Brott, I., de Mink, S. E., Cantiello, M., et al. 2011, A\&A, 530, A115
Butler, K., \& Giddings, J. 1985, Newsletter of Analysis of Astron. Spec., 9 (Univ. London)

Charbonneau, P. 1995, ApJS, 101, 309

Chini, R., Hoffmeister, V. H., Nasseri, A., Stahl, O., \& Zinnecker, H. 2012, MNRAS, 424, 1925

Garmany, C. D., Conti, P. S., \& Massey, P. 1980, ApJ, 242, 1063

Giddings, J. 1981, Ph.D. Thesis, Univ. London

Gies, D. R., Fullerton, A. W., Bolton, C. T., et al. 1994, ApJ, 422, 823

Hadrava, P. 1995, A\&AS, 114, 393

Hadrava, P. 1997, A\&AS, 122, 581

Hadrava, P. 2004, Publ. Astron. Inst. Acad. Sci. Czech Rep., 92, 15

Hadrava, P. 2005, Ap\&SS, 296, 239

Harmanec, P. 1988, BAC, 39, 329

Harmanec, P. 2003, in New Directions for Close Binary Studies: The Royal Road to the Stars, Publ. Canakkale Onsekiz Mart Univ., 3, 221

Herbig, G. H. 1995, ARA\&A, 33, 19

Holmgren, D. E., Hadrava, P., Harmanec, P., et al. 1999, A\&A, 345, 855

Horn, J., Kubát, J., Harmanec, P., et al. 1996, A\&A, 309, 521

Kraus, S., Weigelt, G., Balega, Y. Y., et al. 2009, A\&A, 497, 195

Lanz, T., \& Hubeny, I. 2003, ApJS, 146, 417

Lanz, T., \& Hubeny, I. 2007, ApJS, 169, 83

Linder, N., Rauw, G., Sana, H., De Becker, M., \& Gosset, E. 2007, A\&A, 474, 193

Lucy, L. B., \& Sweeney, M. A. 1971, AJ, 76, 544

Martins, F., Schaerer, D., \& Hillier, D. J. 2005, A\&A, 436, 1049

Nemravová, J., Harmanec, P., Koubský, P., et al. 2012, A\&A, 537, A59

Nieva, M. F., \& Przybilla, N. 2007, A\&A, 467, 295

Nieva, M. F., \& Przybilla, N. 2012, A\&A, 539, A143

Otero, S. A. 2007, OEJV, 72, 1

Pavlovski, K., \& Hensberge, H. 2005, A\&A, 439, 309

Pavlovski, K., \& Southworth, J. 2009, MNRAS, 394, 1519

Pavlovski, K., Tamajo, E., Koubský, P., et al. 2009, MNRAS, 400, 791

Pojmanski, G. 2002, Acta Astron., 52, 397

Polidan, R. S., \& Plavec, M. J. 1984, AJ, 89, 1721

Prša, A., \& Zwitter, T. 2005, ApJ, 628, 426

Prša, A., \& Zwitter, T. 2006, Ap\&SS, 304, 347

Simón-Diaz, S. 2010, A\&A, 510, A22

Škoda, P. 1996, in Astronomical Data Analysis Software and Systems V, ASP Conf. Ser., 101, 187

Stickland, D. J., \& Lloyd, C. 1999, The Observatory, 119, 16

Tamajo, E., Pavlovski, K., \& Southworth, J. 2011, A\&A, 526, A76

Trepl, L., Hambaryan, V. V., Pribulla, T., et al. 2012, MNRAS, 427, 1014

Vaz, L. P. R., Cunha, N. C. S., Vieira, E. F., \& Myrrha, M. L. M. 1997, A\&A, 327,1094

Walborn, N. R. 1972, AJ, 77, 312

Williams, A. M., Gies, D. R., Bagnuolo, Jr., W. G., et al. 2001, ApJ, 548, 425

Zahn, J.-P. 1975, A\&A, 41, 329

Zahn, J.-P. 1977, A\&A, 57, 383 